\title{
A Case of DIPNECH and Review of the Current Literature
}

\author{
Patrick D. Mitchell ${ }^{*}$, Marcus P. Kennedy, Michael T. Henry \\ Cork University Hospital, Cork, The Republic of Ireland \\ Email: "patrickdavidmitchell@gmail.com
}

Received January 24, 2013; revised February 25, 2013; accepted March 3, 2013

Copyright (c) 2013 Patrick D. Mitchell et al. This is an open access article distributed under the Creative Commons Attribution License, which permits unrestricted use, distribution, and reproduction in any medium, provided the original work is properly cited.

\begin{abstract}
Diffuse Idiopathic Neuroendocrine Cell Hyperplasia (DIPNECH) is a rare pulmonary disease. It was first described by Aguayo et al. in 1992, and recognised by the World Health Organisation in 2004 as a precursor lesion to the development of pulmonary carcinoid tumour. DIPNECH has been described in several isolated case reports and series. This article describes a case of DIPNECH and summaries the recent literature in an attempt to raise awareness of this disease and management options.
\end{abstract}

Keywords: Neuroendocrine; Carcinoid; DIPNECH

\section{Case Review}

This case report describes a 73-year-old female with symptoms of intermittent cough, flushing and wheeze for approximately 18 months. A chest x-ray revealed acircumscribed $1.2 \mathrm{~cm}$ opacity in her left upper lobe. She was an ex smoker with a ten year pack history. A contrast thoracic CT scan demonstrated a $1.4 \mathrm{~cm}$ nodule in the left upper lobe biopsied by a CT guided procedure (Figure 1(a)) and multiple other subcentimetre nodules scattered through both lung (Figure 1(b)).

Histological analysis revealed neoplastic neuroendocrine cell proliferation consistent with a carcinoid tumour. She had a PET/CT scan which was reported as normal with no increased FDG uptake detected. She went on to have an elective left upper lobectomy and mediastinal lymphadenectomy.

The surgical specimen was examined and confirmed the diagnosis of a carcinoid tumour but also that of several discrete separate carcinoid tumourlets and the presence of Diffuse Idiopathic Neuroendocrine Cell Hyperplasia (DIPNECH) (Figure 2).

The lymph nodes sampled were benign. She was discharged with regular three monthly follow up and imaging. Unfortunately her symptoms of wheeze, cough and episodic facial flushing failed to subside. A repeat CT thorax demonstrated unchanged sub centimetre nodules as previously described. A Somatostatin labelled scan did

"Corresponding author. not detect any abnormalities suggestive of carcinoid tumour. Biochemical tests including serum Chromogranin A, Chromogranin B and Urinary 5-Hydrxyindolacetic Acid (5-HIAA) were all within normal physiological ranges. She was commenced on a somatostatin analogue Sandostatin ${ }^{\circledR}$ (Novatis, UK), $50 \mathrm{mg}$ given once every four weekssubcutaneously. Her symptoms of wheeze, flushing and diaphoresis abated within weeks of commencing treatment. CT thorax with contrast one year later demonstrated no increase in the size of the lung nodules. This case is a presentation of persistentpulmonary carcinoid syndrome despite the excision of a carcinoid tumour with DIPNECH concomitantly found and treated with a Somatostatin analogue.

\section{Introduction}

Pulmonary neuroendocrine cells are found in very small numbers in the adult lung. These cells produce many physiologically active peptides such as Bombesin, Serotonin, Substance P, Calcitonin and Gastrin. These peptides have been shown to stimulate fibroblasts proliferation, cause bronchoconstriction and have other paracrine effect (6). The World Health Organization (WHO) 2004 Pulmonary Neoplasm classification system formally recognised diffuse idiopathic pulmonary neuroendocrine cell hyperplasia (DIPNECH) as a precursor lesion to the development of pulmonary carcinoid tumour and tumourlets [1,2]. Aguayo et al. was the first to formally de- 


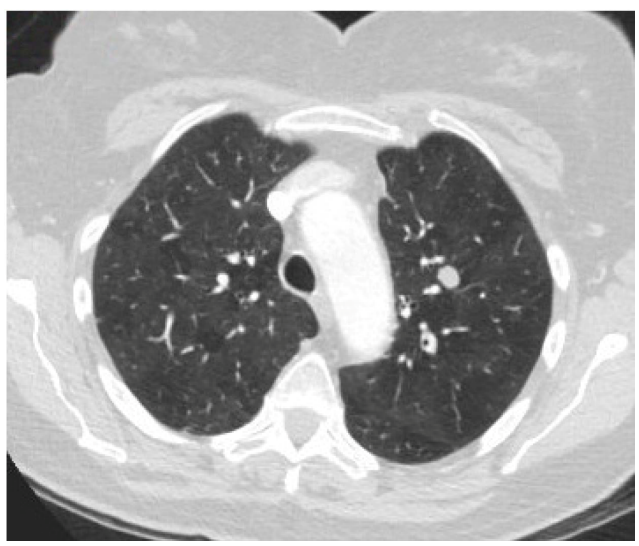

(a)

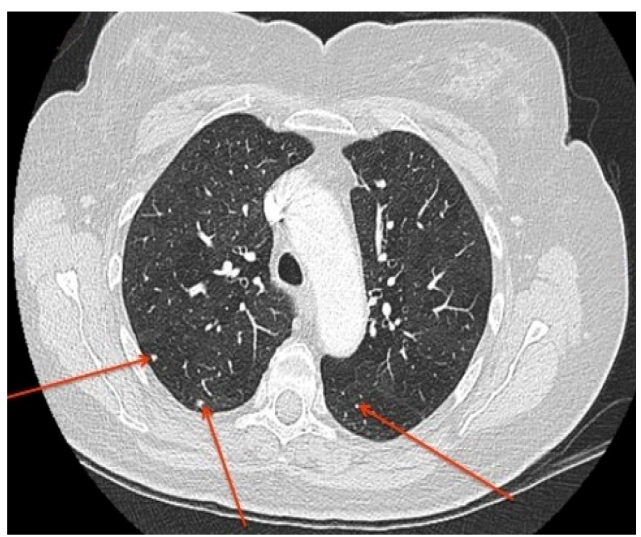

(b)

Figure 1. Axial Contrast Ct thorax of a 73-year-old female with symptoms of intermittent cough, flushing and wheeze for approximately 18 months. (a) Demonstrates the $1.4 \mathrm{~cm}$ left upper lobe nodule; (b) Demonstrates three smaller sub centimetre nodules (red arrows).

scribe this lesion in 1992 in a case series of six patients. In these six patients the authors described diffuse pulmonary neuroendocrine cell hyperplasia in non-smokers with no other lung disease.

\section{Clinical Presentation}

The demographic profile of DIPNECH is limited due to its rarity. From the available case reviews it appears that $90 \%$ of those histologically confirmed have been diagnosed in females with a mean age of 62 (range 56 - 76) [3]. About $67 \%$ of these patients were remote or life-long non-smokers [4]. Of cases diagnosed in one case review $90 \%$ of patients were found to have pulmonary symptoms and the remainder was incidental findings found on surgical biopsies. Symptoms reported included cough (70\%), dyspnoea (63\%) and wheezing (25\%) [3]. In another case series Davis et al. identified 19 patients with histologically confirmed DIPNECH. In this review 10 patients were asymptomatic (8 of these were under surveillance for previously diagnosed cancer) whilst 9 pa-

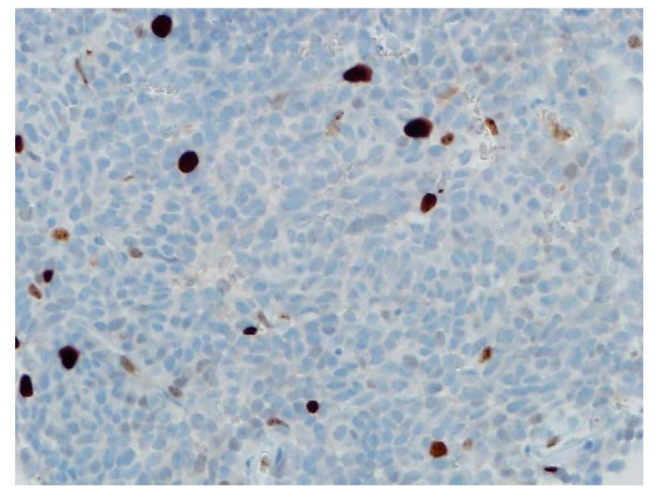

(a)

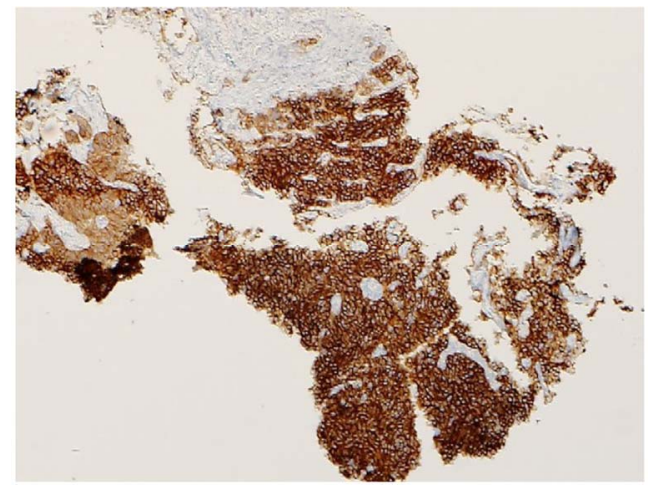

(b)

Figure 2. (a) High power image of the CT guided biopsy showing sparse Ki 67 staining suggestive of a low mitotic turnover; (b) Stained extensively by CD 56 which is found with neuroendocrine tumours.

tients had symptoms of dyspnoea $(n=6)$, cough $(n=4)$, pleuritic chest pain $(n=2)$ and haemoptysis $(n=1)$ [5]. Interestingly there was a striking range from symptom onset to diagnosis of 4 to 16 years (mean of 8.4 years). This lag time suggests a relatively indolent course and explains why it has often been misdiagnosed as asthma. The patient reported in the initial case presentation is the only case described where carcinoid syndrome persisted after the surgical removal of a carcinoid tumour and DIPNECH to our knowledge. Most patients with DIP$\mathrm{NECH}$ have indolent and non-progressive course, with $83 \%$ of patients with symptoms being alive at 5 years [5]. The majority of documented cases of DIPNECH occur in non-smoking middle aged females presenting with cough and obstructive spirometry. The general prognosis of patients with carcinoid tumourlets, probably an advanced form of DIPNECH, has been shown be very good as evidenced by one group reviewing 28 patients post surgical resections which included one case of DIPNECH. In this cohort of 28 patients 3 had died over a six year surveillance period with two deaths following from lung transplantation for progressive obstructive lung disease and the other an unknown cause [6]. Pulmonary function tests (PFTs) demonstrate obstructive defects in about half 
of cases (54\%) but can be restrictive (13\%) or demonstrate a mixed obstructive and restrictive pattern (17\%). In the review by Davies et al. all symptomatic patients had abnormal PFTs. Miller et al. looked at 25 consecutive patients undergoing resection for peripheral carcinoid tumours and found that only $8 \%$ had evidence of airway obstruction associated with neuroendocrine cell hyperplasia [7].

\section{Diagnosis}

Biochemical markers of disease pulmonary neuroendocrine cells derive from the embryological foregut cells. This differentiation is clinically relevant as foregut and hindgut neuroendocrine cells usually lack the enzyme DOPA decarboxylase and are therefore unable to convert 5-hydroxytryptophan (5-HT) to 5-hydroxyindoeacetic acid (5-HIAA), a metabolite excreted in urine which can be a useful marker of carcinoid activity. The usefulness of serum biomarkers in both DIPNECH and pulmonary carcinoid tumour seems to be limited with only isolated case reports of elevated serum Chromogranin A and urinary 5-HIAA levels [5,8]. Radiological imaging has an essential role to play in the diagnosis of DIPNECH. Pulmonary nodules may be identified on Chest X-ray but it doesn't possess the specificity to out rule subtle parenchymal abnormalities found in DIPNECH.High resolution thoracic CT imaging may reveal small sub-centimetre nodules usually below $5 \mathrm{~mm}$ in $60 \%$ of cases. A mosaic attenuation pattern is alsocommonly found and probably represents the underlying histological features of a constrictive bronchiolitis. Ground glass changes, air trapping and in some case bronchiectasis are other described radiological features. CT thorax identifies at least one lung nodule in approximately $60 \%$ of patients, ground-glass attenuation in 30\% patients, bronchiectasis in about $20 \%$ of patients and mosaic pattern in $17 \%$ in another review [9]. None of these abnormalities are pathognomic. This should prompt further appropriate investigation.

The role of bronchoscopy in DIPNECH has been described. Bronchoscopic inspection, bronchioalveolar lavage (BAL) and both endobronchial and transbronchial biopsies are recommended to rule out other respiratory conditions [10]. BAL results have been found to have a lymphocytosis of around $30 \%$ in two confirmed cases of DIPNECH. A BAL lymphocytosis of $30 \%$ has been described in two cases of DIPNECH REF However the paucity of published experience with bronchoscopy and DIPNECH makes it largely conjecture to comment further. Like wise endobronchial biopsies are felt to have a limited diagnostic role in this condition. This is due to the large biopsy sample needed to diagnose DIPNECH. There are several cases reports of DIPNECH diagnosed via transbronchial biopsy. Nasser et al. points out from a literature review that the yield from transbronchial biopsy is only about $12 \%$ in diagnosing DIPNECH. This is due to the large amount of tissue necessary to pathologically characterize tumourlet and DIPNECH. The gold standard for diagnosing DIPNECH is a surgical lung biopsy [11]. Sampling error will occur if insufficient numbers of airways are included in a biopsy. Diagnosis also relies heavily on histologic specimens being prepared correctly in addition to an experienced lung histopathologist. Nasser et al. reported that $88 \%$ of cases required a surgical biopsy to confirm the diagnosis [12]. The pathological diagnosis of DIPNECH is reserved only for cases in which there is neuroendocrine cell hyperplasia confined to airway epithelium. These proliferations are superficial to the basement membrane and confined to the bronchial and bronchiolar epithelium unlike carcinoid tumourlets which invade the basement. There is wide- spread proliferation of pulmonary neuroendocrine cells which can manifest as increased numbers of individual cells, small groups, or nodular aggregates or nests in the bronchial or bronchiolar epithelium is the hallmark of DIPNECH (Figure 3).

Reactive pulmonary neuroendocrine cell hyperplasia is common but a separate entity to DIPNECH. One study compared the morphology and antigenic profile of pulmonary neuroendocrine cells (PNECs) proliferating as a reaction to pulmonary injury with those proliferating in diffuse idiopathic pulmonary neuroendocrine cell hyperplasia (DIPNECH) in which carcinoids develop. In this study there were differences between the groups in expression of p53, p16 and Ki67. These are cell proliferation antigens. These antigens were seen more consistently and earlier in DIPNECH than reactive pulmonary neuroendocrine cell hyperplasia. CD56, chromogranin,

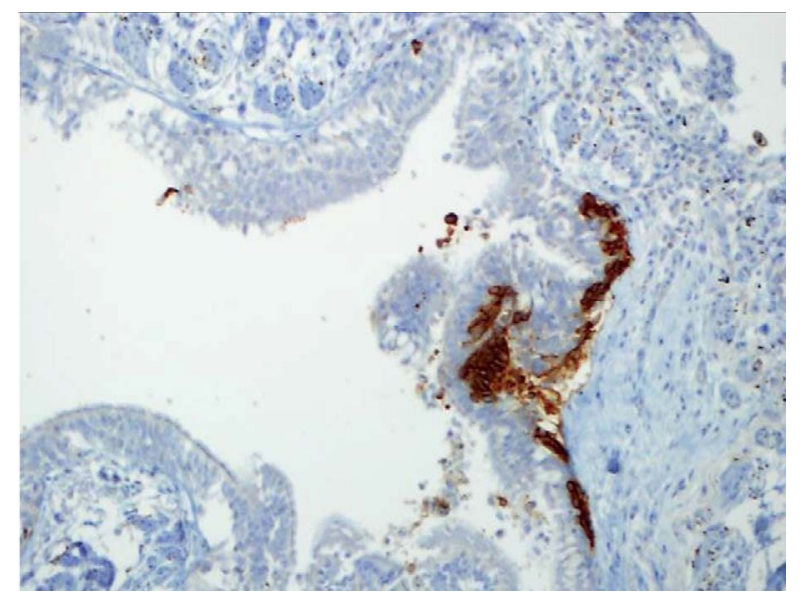

Figure 3. This a low power picture of a synaptophysinimmunohistochemical stain which shows nicely a group of positively staining neuroendocrine cells (brown) confined to bronchiolar epithelium not penetrating through the basement membrane which is the classic appearance of DIPNECHmembrane (see Figure 3). 
synaptophysin are the immunohistochemical markers of Neuroendocrine Cells. A Ki-67 index is used as a marker of cell proliferation [13].

Nasser et al. again reported that $40 \%$ of cases of confirmed DIPNECH there was a concomitant carcinoid tumour and $70 \%$ of cases had concomitant carcinoid tumourlets [12]. Pulmonary neuroendocrine cells commonly produce bombesin and gastrin-releasing peptides [14]. These amine peptides stimulate fibroblasts and bronchoconstriction of airway cells which may lead to peribronchiolar and interstitial fibrosis accounting for symptoms and spirometric abnormalities [14]. DIPNECH stains nearly universally for EGFR (cells exhibited a maximum EGFR expression score of 3 in $100 \%$ of cells) and in contrast $90 \%$ of large cell neuroendocrine and small cell neuroendocrine carcinomas were negative for EGFR. This may have certain clinical ramifications as well as a potential pathophysiological role [15]. In another case report DIPNECH found in association with an invasive lung adenocarcinoma DIPNECH has been described in association with an invasive lung adenocarcinoma but this case appears isolated [16].

\section{Management}

Currently there are no consensus led guidelines for the management of DIPNECH. Essentially the evidence for treatment is based upon case reports and small case series and the follow up of these patients. Swigris et al. presented 4 patients and found an excellent long-term prognosis over 14 years [4]. Reviewing of the literature suggests that current therapeutic options have remained focused on steroid-based therapies or Somatostatin analogues in symptomatic patients [14,17-19]. The possible explanationfor the effectiveness of steroids would be the blunting of the inflammatory response stimulated by the neuropeptide secretions from neuroendocrine cell groups [14]. If DIPNECH is histologically confirmed options may include observational "watch and wait" policy or trial of inhaled or systemic corticosteroid therapy with bronchodilator therapy particularly in patients who demonstrate reversible airflow obstruction [12,20,21]. Patients with severe refractory respiratory diseasemay ultimately are referred need to be referred for lung transplant evaluation [4]. Chemotherapy in one small case review of two patients did not have favourable results with one patient ultimately died from progressive respiratory failure while on treatment with fluorouracil [22]. In patients in who are symptomatic with carcinoid symptoms, somatostatin analogues and interferon $\alpha$ have been proposed as tumourostatic agents $[17,18,23,24]$. The patient described in the introductory case study to this review was treated with a lung resection and with a Somatostatin analogue and responded to this therapy with a significant decrease in wheeze, flushing and diarrhoea and stability in the appearance of sub centimetre pulmonary nodules on follow up chest imaging. Gorschtein et al. retrospectively reported on 11 patients, all female, with both carcinoid lung tumour and DIPNECH who were treated with lung resection and a somatostatin analogue. All patients were alive at follow up of a mean of 4.5 years $[10,24]$. There are also reports of clinical endocrinopathies in patients with DIPNECH and include acromegaly and pituitary adenoma, MEN1 syndrome and parathyroid gland hyperplasia with lung tissue immunohistochemical staining positive for GHRH and ACTH [19]. This may relate to its neuroendocrine origins.

\section{Conclusion}

DIPNECH is a rare clinical entity and its diagnosis requires a high level of suspicion with the available correlating clinical and radiological findings. Several diagnosis of DIPNECH have made inadvertently through surgical biopsy. Again, to make the diagnosis a surgical lung biopsy is regarded as the gold standard. The management of patients with DIPNECH remains controversial but several treatments including surgery, steroids and somatostatin analogues are frequently successful. Further studies with somatostatin analogue and steroid therapies in patients with DIPNECH who have evidence of hormonal syndromes or obstructive spirometry are certainly warranted. Given its rarity a multi-centred registry should be established to further guide clinicians and patients.

\section{REFERENCES}

[1] W. D. Travis, E. Brambilla, H. K. Muller-Hermlink and C. C. Harris, "Pathology and Genetics of Tumours of the Lung, Pleura, Thymus and Heart,” In: World Health Organization Classification of Tumours, Lyon, 2004.

[2] K. M. Kerr, "Pulmonary Preinvasiveneoplasia,” Journal of Clinical Pathology, Vol. 54, No. 4, 2001, pp. 257-271. doi:10.1136/jcp.54.4.257

[3] M. G. Carmichael and L. L. Zacher, "The demonstration of Pulmonary Neuroendocrine Cell Hyperplasia with Tumorlets in a Patient with Chronic Cough and a History of Multiple Medical Problems,” Military Medicine, Vol. 170, No. 5, 2005, pp. 439-441.

[4] J. Swigris, S. Ghamande, T. Rice and C. Farver, "Diffuse Idiopathic Neuroendocrine Cell Hyperplasia. An Interstitial Lung Disease with Airway Obstruction,” J Bronch, Vol. 12, 2005, pp. 62-64. doi:10.1097/01.laboratory.0000147030.83001.3c

[5] S. J. Davies, J. R. Gosney, D. M. Hansell, A. U. Wells, R. M. du Bois, M. M. Burke, M. N. Sheppard and A. G. Nicholson, "Diffuse Idiopathic Pulmonary Neuroendocrine Cell Hyperplasia: An Under-Recognised Spectrum of Disease,” Thorax, Vol. 62, No. 3, 2007, pp. 248-252. doi:10.1136/thx.2006.063065

[6] M. C. Aubry, C. F. Thomas Jr., J. R. Jett, S. J. Swensen 
and J. L. Myers, "Significance of Multiple Carcinoid Tumors and Tumorlets in Surgical Lung Specimens: Analysis of 28 Patients," Chest, Vol. 131, No. 6, 2007, pp. 1635-1643. doi:10.1378/chest.06-2788

[7] R. R. Miller and N. L. Muller, "Neuroendocrine Cell Hyperplasia and Obliterative Bronchiolitis in Patients with Peripheral Carcinoid Tumors," American Journal of Surgical Pathology, Vol. 19, No. 6, 1995, pp. 653-658. doi:10.1097/00000478-199506000-00005

[8] E. Seregni, L. Ferrari, E. Bajetta, A. Martinetti and E. Bombardieri, "Clinical Significance of Blood Chromogranin a Measurement in Neuroendocrine Tumours," Annals of Oncology, Vol. 12, Suppl. 2, 2001, pp. S69-S72. doi:10.1093/annonc/12.suppl_2.S69

[9] J. S. Lee, K. K. Brown, C. Cool and D. A. Lynch, "Diffuse Pulmonary Neuroendocrine Cell Hyperplasia: Radiologic and Clinical Features,” Journal of Computer Assisted Tomography, Vol. 26, No. 2, 2002, pp. 180-184. doi:10.1097/00004728-200203000-00003

[10] A. Gorshtein, D. Gross, D. Barak, Y. Strenov, Y. Refaeili, I. Shimon and S. Grozinsky-Glasinshy, "Diffuse Idiopathic Pulmonary Neuroendocrine Cell Hyperplasia and the Associated Lung Neuroendocrine Tumours: Clinical Experience with a Rare Entity," Cancer, Vol. 118, No. 3, 2012, pp. 612-619. doi:10.1002/cncr.26200

[11] S. Dacic, "Pulmonary Preneoplasia," Archives of Pathology \& Laboratory Medicine, Vol. 132, No. 7, 2008, pp. 1073-1078.

[12] A. Nasser, D. Jaroszewski, R. Helmers and T. Colby, "Diffuse Idiopathic Pulmonary Neuroendocrine Cell Hyperplasia: A Systematic Overview," American Journal of Respiratory and Critical Care Medicine, Vol. 184, No. 18-16, 2011, pp. 8-16.

[13] J. R. Gosney, I. J. Williams, A. R. Dodson and C. S. Foster, "Morphology and Antigen Expression Profile of Pulmonary Neuroendocrine Cells in Reactive Proliferations and Diffuse Idiopathic Pulmonary Neuroendocrine Cell Hyperplasia (DIPNECH)," Histopathology, Vol. 59, No. 4, 2011, pp. 751-762. doi:10.1111/j.1365-2559.2011.03992.x

[14] S. Degan, G. Y. Lopez, K. Kevill and M. E. Sunday, "Gastrin-Releasing Peptide, Immune Responses, and Lung Disease," Annals of the New York Academy of Sciences, Vol. 1144, 2008, pp. 136-147. doi:10.1196/annals.1418.022

[15] EGFR, "Expression in Pulmonary Neuroendocrine Cell Hyperplasia,” Pathologe, Vol. 27, No. 2, 2006, pp. 147-
151. doi:10.1007/s00292-006-0819-0

[16] A. Warth, E. Herpel, A. Schmähl, K. Storz and P. A. Schnabel, "Diffuse Idiopathic Pulmonary Neuroendocrine Cell Hyperplasia (DIPNECH) in Association with an Adenocarcinoma: A Case Report," Journal of Medical Case Reports, Vol. 2, 2008, p. 21. doi:10.1186/1752-1947-2-21

[17] L. Salz, "Octreotide as an Antineoplastic Agent in the Treatment of Functional and Nonfunctional Neuroendocrine Tumors,” Cancer, Vol. 72, No. 1, 1993, pp. 244-248. doi:10.1002/1097-0142(19930701)72:1<244::AID-CNCR 2820720143>3.0.CO;2-Q

[18] J. Chan and M. Kulke, "Progress in the Treatment of Neuroendocrine Tumors," Current Oncology Reports, Vol. 11, No. 3, 2009, pp. 193-199. doi:10.1007/s11912-009-0028-0

[19] M. B. Fessler, C. D. Cool, Y. E. Miller, M. I. Schwarz and K. K. Brown, "Idiopathic diffuse Hyperplasia of Pulmonary Neuroendocrine Cells in a Patient with Acromegaly,” Respirology, Vol. 9, No. 2, 2004, pp. 274-277. doi:10.1111/j.1440-1843.2004.00571.X

[20] M. S. Sanaee, P. M. O’Byrne and P. Nair, "Diffuse Idiopathic Pulmonary Neuroendocrine Hyperplasia, Chronic Eosinophilic Pneumonia, and Asthma,” European Respiratory Journal, Vol. 34, No. 6, 2009, pp. 1489-1492. doi:10.1183/09031936.00109409

[21] E. N. Coletta, L. R. Voss, M. S. Lima, J. S. Arakaki, J. Camara, C. D'Andretta Neto and C. A. Pereira, "Diffuse Idiopathic Pulmonary Neuroendocrine Cell Hyperplasia Accompanied by Airflow Obstruction,” Jornal Brasileiro de Pneumologia, Vol. 35, No. 5, 2009, pp. 489-494.

[22] S. M. Aguayo, Y. E. Miller, J. A. Waldron Jr., R. M. Bogin, M. E. Sunday, G. W. Staton Jr, W. R. Beam and T. E. King Jr., "Brief Report: Idiopathic Diffuse Hyperplasia of Pulmonary Neuroendocrine Cells and Airways Disease," New England Journal of Medicine, Vol. 327, 1992, pp. 1285-1288. doi:10.1056/NEJM199210293271806

[23] L. Saltz, "Octreotide as an antineoplastic Agent in the Treatment of Functional and Non-Functional Neuroendocrine Tumours," Cancer, Vol. 72, No. 1, 1993, pp. 244-248. doi:10.1002/1097-0142(19930701)72:1<244::AID-CNCR 2820720143>3.0.CO;2-Q

[24] J. Chan and M. Kulke, "Progress in the Treatment of Neuroendocrine Tumors,” Current Oncology Reports, Vol. 11, No. 3, 2009, pp. 193-199. doi:10.1007/s11912-009-0028-0 